\title{
Interface entre saúde mental e pandemia da COVID-19: o olhar dos profissionais de
}

\section{saúde}

\author{
Interface between mental health and the COVID-19 pandemic: the perspective of health \\ professionals
}

Interfaz entre la salud mental y la pandemia de COVID-19: la perspectiva de los profesionales de la salud

\author{
Gabriela da Silva Gonçalves \\ ORCID: https://orcid.org/0000-0003-4118-3379 \\ Universidade Federal de Juiz de Fora, Brasil \\ E-mail: gabrielamef@gmail.com \\ Fernanda de Oliveira Ferreira \\ ORCID: https://orcid.org/0000-0002-5857-2824 \\ Universidade Federal de Juiz de Fora, Brasil \\ E-mail: fernanda.ferreira@ufjf.edu.br \\ Vanessa Monteiro de Melo Sousa \\ ORCID: https://orcid.org/0000-0003-4111-3784 \\ Universidade Federal de Juiz de Fora, Brasil \\ E-mail vanessamonteiromelo92@gmail.com \\ Maria Helena Braga \\ ORCID: https://orcid.org/0000-0002-2786-6977 \\ Universidade Federal de Juiz de Fora, Brasil \\ E-mail: maria.helena@ufjf.edu.br \\ Terezinha Noemides Alves \\ ORCID https://orcid.org/0000-0003-3674-8267 \\ Universidade Federal de Juiz de Fora, Brasil \\ E-mail: noemides@uol.com.br \\ Patrícia Aparecida Baumgratz de Paula \\ ORCID: https://orcid.org/0000-0001-7769-7772 \\ Universidade Federal de Juiz de Fora, Brasil \\ E-mail: patricia.paula@ufjf.edu.br
}

\begin{abstract}
Resumo
A saúde mental tem sido marcada atualmente por diversas reformulações, incluindo a intensificação da medicalização da saúde e presença da pandemia do novo coronavírus. Este trabalho teve como objetivo descrever a percepção da saúde mental pelos profissionais dos programas de residência em saúde, em 2020, durante a pandemia da COVID-19. Tratou-se de estudo observacional e transversal, com abordagem quantitativa, com aplicação de questionário estruturado, auto aplicado e online, para 52 residentes dos Programas de Residência Uni e Multiprofissionais do Hospital Universitário da Universidade Federal de Juiz de Fora. Os dados obtidos foram trabalhados pela análise estatística descritiva e o teste qui-quadrado para verificar a associação entre a autopercepção da saúde mental, as variáveis sociodemográficas e o perfil profissional. Com o intuito de verificar o efeito da realização do acompanhamento da saúde mental, realizou-se análise de regressão logística. Os resultados demonstraram agravamento significativo na percepção da saúde física e mental pelos residentes, comparando o período antes e durante a pandemia, em 2020, e que o acompanhamento da saúde mental foi fator protetor para alterações fisiológicas percebidas pelos residentes no período. Assim, o apoio psicossocial aos profissionais de saúde é de fundamental importância, principalmente quando consideramos o cenário pandêmico.
\end{abstract}

Palavras-chave: Saúde mental; Pandemia; COVID-19; Profissionais de saúde.

\begin{abstract}
Mental health has currently been marked by several reformulations, including the intensification of the health medicalization and the new coronavirus pandemic. This work aimed to describe the perception of mental health by professionals of residency programs in health, in 2020, during the COVID-19 pandemic. It's an observational and transversal study, with a quantitative approach. We have done a structured, self-applied and online questionnaire, for 52 residents of the Uni and Multi Professional Residency Programs at the Universitary Hospital of the Federal University of Juiz de Fora. The obtained data were processed using as analysis method descriptive statistics and the chi-square test to verify the association between self-perception of mental health, sociodemographic variables and
\end{abstract}


professional profile. In order to verify the effect of mental health care, logistic regression analysis was performed. The results showed a significant worsening in the perception of physical and mental health by residents, comparing the period before and during the pandemic in 2020, and that the previous and continuous mental health care was a protective factor for physiological changes perceived by residents in the period. Thus, psychosocial support to health professionals is essential, especially when we consider the pandemic scenario.

Keywords: Mental health; Pandemic; COVID-19; Health professionals.

\section{Resumen}

La salud mental ha estado marcada actualmente por varias reformulaciones, entre ellas la intensificación de la medicalización de la salud y la presencia de la nueva pandemia de coronavirus. Este estudio tuvo como objetivo describir la percepción de la salud mental por parte de los profesionales en programas de residencia en salud, en 2020 , durante la pandemia de COVID-19. Se trata de un estudio observacional y transversal, con enfoque cuantitativo, con la aplicación de un cuestionario estructurado, autoadministrado y en línea a 52 residentes de los Programas de Residencia Uni y Multiprofesional del Hospital Universitario de la Universidad Federal de Juiz de Fora. Los datos se analizaron mediante análisis estadístico descriptivo y la prueba de chi-cuadrado para verificar la asociación entre la autopercepción de la salud mental, las variables sociodemográficas y el perfil profesional. Para verificar el efecto del monitoreo de la salud mental, se realizó un análisis de regresión logística. Los resultados mostraron un deterioro significativo en la percepción de la salud física y mental de los residentes, comparando el período anterior y durante la pandemia, en 2020, y que el monitoreo de la salud mental fue un factor protector de los cambios fisiológicos percibidos por los residentes en el período. Por lo tanto, el apoyo psicosocial a los profesionales de la salud es de fundamental importancia, especialmente cuando se considera el escenario pandémico.

Palabras clave: Salud mental; Pandemia; COVID-19; Profesionales de la salud.

\section{Introdução}

A atenção à saúde mental tem passado por profundas transformações, tanto no cenário mundial quanto no nacional, no tocante à superação do modelo manicomial (Campos, 2019).

Durante a década de 1980 no Brasil, o campo da saúde foi marcado pelo reconhecimento do direito à saúde enquanto atributo de cidadania. No tocante à saúde mental, o movimento da reforma psiquiátrica tornou-se parte integrante do processo de redemocratização do país, com ênfase na desinstitucionalização.

Nesse sentido, cabem destacar a realização, em 1987, da $1^{\text {a }}$ Conferência Nacional de Saúde Mental (CNSM), como um dos desdobramentos da $8^{\text {a }}$ Conferência Nacional de Saúde realizada no ano de 1986, e a Constituição Federal de 1988 ao criar o Sistema Único de Saúde (SUS), com seus princípios e diretrizes: universalidade, equidade e integralidade das ações e serviços de saúde (Guedes et al., 2021).

Nos anos de 1990, em paralelo à política de saúde adotada, houve aprovação de aparatos regulatórios em relação ao financiamento da saúde mental e ao funcionamento dos serviços psiquiátricos. Em 1992, foi realizada a $2^{\mathrm{a}}$ CNSM que apresentou uma nova lógica de organização dos serviços de saúde mental, com foco nos usuários destes, como portadores de direitos (Sy, 2018).

Ao dar continuidade a tais discussões, a Política Nacional de Saúde Mental (PNSM) foi aprovada pela Lei $\mathrm{n}^{\circ}$ 10.216/2001, que propôs a criação de práticas e serviços comunitários que substituíssem hospitais psiquiátricos. Logo, a PNSM possibilitou a evolução na forma de percepção do sofrimento psíquico, contrapondo-se à lógica manicomial de controle do tempo e da mente dos indivíduos, evidenciando a reabilitação psicossocial como um novo modelo na configuração do cuidado em saúde mental2. Em 2010, houve a realização da $4^{\mathrm{a}} \mathrm{CNSM}$ visando ao delineamento estratégico de políticas públicas para atenção à saúde mental e enfatizando a abordagem intersetorial (Sy, 2018).

Dentro desse contexto e após 20 anos da criação da PNSM, alguns desafios ainda se fazem presentes no campo da saúde mental, dentre eles: a dificuldade de se estabelecer a intersetorialidade, o acesso aos serviços de saúde, a ampliação do número de leitos psiquiátricos em hospitais gerais, a presença de equipes bem preparadas para o atendimento dos usuários, além da disseminação do crack (Sy, 2018).

Acrescido a esse cenário, na sociedade brasileira contemporânea têm-se a medicalização da saúde e a pandemia do 
novo coronavírus (COVID-19).

$\mathrm{Na}$ área da saúde mental, a medicalização também assume relevância e gravidade, especialmente por se constituir um campo de disputas de modelos de cuidado em que a racionalidade psiquiátrica revela forte poder2. Diversos processos naturais da vida cotidiana, tais como: vivência da sexualidade, parto, desenvolvimento infantil, menopausa, desconforto menstrual, envelhecimento, morte, conflitos familiares e interpessoais, dificuldades de aprendizagem, entre outros, passaram a ser medicalizados, o que configura a medicalização da vida (Schechtman, 2018).

Nessa perspectiva, a pandemia do novo coronavírus tornou-se um desafio de grande envergadura para o campo da saúde mental, como emergência de saúde pública mundial e brasileira. Essa pandemia traz preocupações relacionadas à saúde física, à saúde mental e ao sofrimento que podem ser vivenciados pelos diversos atores sociais: população em geral, usuários, profissionais da saúde e prestadores de serviços, entre outros.

Estudos sobre as implicações na saúde mental em decorrência da pandemia do novo coronavírus apontam para repercussões negativas importantes, principalmente quando se leva em consideração os profissionais de saúde. Estes costumam vivenciar situações de estresse no contexto de pandemias, dentre elas: risco aumentado de ser infectado, de adoecer e até de morrer; possibilidade de, inadvertidamente, infectar outras pessoas; sobrecarga e fadiga; exposição a mortes em larga escala, entre outras vivências (Schmid et al., 2020).

Portanto, essas mudanças nas práticas de trabalho impactaram direta ou indiretamente nos serviços de assistência à saúde mental, o que evidencia a urgência e relevância sobre a percepção da saúde mental por um grupo de profissionais dos programas de residência em saúde, na pandemia da COVID-19, objeto do presente estudo.

\section{Metodologia}

Tratou-se de uma abordagem quantitativa, utilizando o estudo observacional e transversal. Segundo Mussi e colaboradores (2019), a abordagem quantitativa caracteriza-se por não se interessar pelo singular, o individual, o diferenciado, ou seja, o pessoal. Neste tipo de abordagem, o interesse é no coletivo, naquilo que pode ser predominante como característica do grupo.

No campo da saúde, as pesquisas mais frequentes são feitas na forma de estudos observacionais. Nesse tipo de investigação científica os pesquisadores não interferem nos fenômenos em estudo, coletam dados para proceder a sua descrição e/ou análise. Assim, os estudos de corte transversal visam obter dados fidedignos que ao final da pesquisa permitam elaborar conclusões confiáveis, robustas, além de gerar novas hipóteses que poderão ser investigadas com novas pesquisas (Raimundo et al, 2018).

Nesse sentido, no período compreendido entre outubro a dezembro de 2020, foram aplicados questionários estruturados aos 52 residentes dos Programas de Residência Uni e Multiprofissionais do Hospital Universitário (HU) da Universidade Federal de Juiz de Fora (UFJF), das seguintes áreas profissionais: Administração, Economia, Enfermagem, Educação Física, Farmácia (incluindo Análises Clínicas), Fisioterapia, Nutrição, Odontologia, Psicologia e Serviço Social. Esses programas englobam as Residências em Saúde do Adulto, em Saúde Mental, em Saúde da Família, em Atenção Hospitalar, Uni profissional em Farmácia e em Gestão Hospitalar.

Esses questionários foram respondidos on line por meio do formulário do Google Forms, com ênfase nas seguintes variáveis categóricas: Perfil socioeconômico e demográfico; Perfil profissional; Autopercepção de saúde.

O tratamento estatístico dos dados obtidos foi realizado por meio da análise estatística descritiva (distribuição de frequência). Na análise descritiva foram determinadas as frequências absolutas e percentuais. Para verificar a associação entre a autopercepção de saúde mental e as variáveis sociodemográficas e características de perfil profissional foi utilizado o teste qui-quadrado. 
Com o intuito de verificar o efeito da realização de um acompanhamento de saúde mental, foi realizada uma análise de regressão logística, considerando como variável independente a realização de acompanhamento de saúde mental previamente à pandemia e, como desfechos, as ocorrências das variáveis cansaço extremo, falta de concentração, alteração de sono e alterações de apetite presentes ou não durante a pandemia. A seleção das variáveis independentes baseou-se no critério de ter apresentado valor $\mathrm{p}<0,20$ nas análises bivariadas.

Todos os resultados foram considerados estatisticamente significativos ao nível de 5\%. O software utilizado na análise foi o Statistical Package for the Social Sciences (SPSS), versão 20.0 para Windows.

O presente estudo teve aprovação do Comitê de Ética em Pesquisa (CEP) da UFJF, sob o n ${ }^{\circ} 4.342 .755$.

\section{Resultados}

\subsection{Perfil socioeconômico, demográfico e profissional}

Para facilitar a visualização dos dados socioeconômicos, demográficos e profissionais, as variáveis quantitativas serão apresentadas agrupadas.

A média de idade é de 26 anos, com predomínio do sexo feminino (88,5\%), sendo 98,1\% do gênero Cis sexual, isto é, se identifica com o sexo biológico com o qual nasceu e 1,9\% que preferiu não informar. Quanto à orientação sexual, 82,7\% são heterossexuais, $9,6 \%$ homossexuais e 7,7\% bissexuais. Dos entrevistados, $65,4 \%$ se declaram brancos, $28,8 \%$ pardos e $5,8 \%$ pretos; além de ter sido declarado que $90,4 \%$ não possuem filhos.

Além disso, 42,3\% dos participantes residem com a família e 26,9\% com companheiro (a). Em relação ao COVID-19, 57,7\% não moram com alguém considerado como grupo de risco. Quanto à renda, 50\% possuem renda familiar entre 3 a 4 salários mínimos, sem considerar os valores financeiros das bolsas de residência, como auxílio financeiro oficial, recebidos pelos entrevistados. Também foi evidenciado que 60,8\% vivem em imóvel alugado.

Em relação ao perfil profissional, destaca-se que 40,4\% são residentes do Programa de Residência Multiprofissional em Saúde do Adulto; 32,7\% da Integrada Multiprofissional em Atenção Hospitalar; 11,5\% da Multiprofissional em Saúde da Família; 3,8\% da Multiprofissional em Saúde Mental; 3,8 \% da Farmácia e 7,7 \% da Gestão Hospitalar.

Quanto à jornada de trabalho durante a pandemia, $61,5 \%$ dos residentes responderam que a carga horária semanal de 60 horas de trabalho foi mantida normalmente (48 horas de prática no cenário e 12 horas de carga teórica).

No tocante aos cenários de atuação, 66,7\% dos residentes atuavam no Hospital Universitário da UFJF e 29,4\% em Unidades Básicas de Saúde, entre outros locais de atuação. Cabe ainda destacar que 49\% dos residentes avaliaram como sendo alta a exposição ao COVID-19 no seu ambiente de trabalho. Sobre o nível de segurança (suporte, o uso de Equipamento de Proteção Individual (EPI) e a organização do fluxo laboral) do ambiente de trabalho com relação à possibilidade de contaminação é considerado médio por $64,7 \%$ dos residentes.

\subsection{Autopercepção de saúde}

Nas análises de associação realizadas não foi observada nenhuma associação significativa entre as variáveis socioeconômicas e demográficas, além da percepção da saúde física e mental ( $p>0,05)$.

Houve um agravamento significativo na percepção da saúde física $(\mathrm{p}=0.003)$ e mental $(\mathrm{p}<0.001)$ pelos residentes, comparando o período antes e durante a pandemia, em 2020, como demonstra a Tabela 1. 
Tabela 1. Percepção da saúde física e mental pelos residentes antes e durante a pandemia, em 2020.

\begin{tabular}{ccccccccccc}
\hline & \multicolumn{4}{c}{ Percepção da Saúde Física } & \multicolumn{3}{c}{ Percepção da Saúde Mental } \\
& $\begin{array}{c}\text { Muito } \\
\text { boa }\end{array}$ & Boa & Razoável & Ruim & $\begin{array}{c}\text { Muito } \\
\text { ruim }\end{array}$ & $\begin{array}{c}\text { Muito } \\
\text { boa }\end{array}$ & Boa & $\begin{array}{c}\text { Razoável } \\
\text { Ruim }\end{array}$ & $\begin{array}{c}\text { Muito } \\
\text { ruim }\end{array}$ \\
\hline Antes da & $11,5 \%$ & $65,4 \%$ & $23,1 \%$ & - & - & $5,8 \%$ & $69,2 \%$ & $21,2 \%$ & $3,8 \%$ & - \\
Pandemia & $(6)$ & $(34)$ & $(12)$ & & & $(3)$ & $(36)$ & $(11)$ & $(2)$ & \\
Durante a & $3,8 \%$ & $23,1 \%$ & $55,8 \%$ & $15,4 \%$ & $1,9 \%$ & - & $13,5 \%$ & $44,2 \%$ & $34,6 \%$ & 7,7 \\
Pandemia & $(2)$ & $(12)$ & $(29)$ & $(8)$ & $(1)$ & & $(7)$ & $(23)$ & $(18)$ & $\%(4)$ \\
P & & & 0,003 & & & & & $<0,001$ & \\
\hline
\end{tabular}

Fonte: Autores.

Quanto à ocorrência de sintomas e à realização do acompanhamento em saúde mental, pode-se perceber que as frequências de alterações de sono $(p=0,02)$, alterações de apetite $(p=0,028)$, falta de concentração $(p=0,03)$ e de cansaço extremo $(\mathrm{p}=0,03)$ foram mais elevadas nos residentes que não realizam acompanhamento em saúde mental, conforme demonstrado na Tabela 2.

Tabela 2. Associação entre a ocorrência de sintomas e acompanhamento em saúde mental.

\begin{tabular}{lccc}
\hline Alteração & Realiza acompanhamento em saúde mental & \multicolumn{2}{c}{ Valor de p } \\
& Não \% (n) & Sim \% (n) & 0,023 \\
\hline Frequência de alterações de sono (Sim) & $72,4 \%(21)$ & $61,1 \%(11)$ & 0,028 \\
Frequência de alterações de apetite (Sim) & $75,0 \%(18)$ & $56,5 \%(13)$ & 0,030 \\
Frequência de falta de concentração (Sim) & $69,7 \%(23)$ & $64,3 \%(9)$ & 0,027 \\
Frequência de cansaço extremo (Sim) & $71,0 \%(22)$ & $62,5 \%(10)$ & \\
\hline
\end{tabular}

Legenda: p: frequência de alterações. Fonte: Autores.

A análise de regressão logística indicou que o acompanhamento de saúde mental foi fator protetor para alterações fisiológicas percebidas pelos residentes durante a pandemia (cansaço extremo, falta de concentração, alterações de sono e apetite), conforme Tabela 3 .

Tabela 3. Associação entre a realização de acompanhamento em saúde mental e cansaço extremo, falta de concentração, alterações de sono e apetite.

\begin{tabular}{|c|c|c|c|c|c|c|c|c|c|c|c|c|}
\hline \multirow{2}{*}{$\begin{array}{c}\text { Realiza } \\
\text { acompanha-mento em } \\
\text { saúde mental }\end{array}$} & \multicolumn{3}{|c|}{ Cansaço Extremo } & \multicolumn{3}{|c|}{ Falta de concentração } & \multicolumn{3}{|c|}{ Alteração de sono } & \multicolumn{3}{|c|}{ Alteração de apetite } \\
\hline & OR & IC $95 \%$ & $\mathrm{p}$ & OR & IC $95 \%$ & $\mathrm{p}$ & OR & IC 95\% & $\mathrm{p}$ & OR & IC $95 \%$ & $\mathrm{p}$ \\
\hline & 0,245 & $\begin{array}{c}0,069- \\
0,878\end{array}$ & 0,031 & 0,242 & $\begin{array}{c}0,064- \\
0,906\end{array}$ & 0,035 & 0,242 & $\begin{array}{c}0,069- \\
0,846\end{array}$ & 0,026 & 0,256 & $\begin{array}{c}0,074- \\
0,885\end{array}$ & 0,031 \\
\hline
\end{tabular}

Legenda: OR (odds ratio) - Razão de Chance; IC (Intervalo de Confiança); p (frequência das alterações fisiológicas). Fonte: Autores.

Os dados indicaram que a realização de acompanhamento de saúde mental reduz em $75 \%$ a probabilidade de cansaço 
extremo $(\mathrm{OR}=0,245)$, em 76\% a chance de falta de concentração $(\mathrm{OR}=0,242)$, em $74 \%$ a probabilidade de alteração de apetite $(\mathrm{OR}=0,256)$ e em $76 \%$ a probabilidade de alteração de sono $(\mathrm{OR}=0,242)$.

Além disso, ao considerar o acesso ao serviço de saúde mental durante a pandemia, cabe destacar que: os residentes das áreas de Saúde da Família (83,3\%), Saúde do Adulto (61,9\%) e Saúde Mental (54,2\%) foram os que mais procuraram esse serviço. Já os residentes da Residência Multiprofissional em Atenção Hospitalar (76,5\%) procuraram em menor quantidade e os residentes da área de Gestão Hospitalar não procuraram o serviço de saúde mental, conforme a Tabela 4.

Tabela 4. Acesso ao serviço de saúde mental durante a pandemia.

\begin{tabular}{cc}
\hline Área de Residência & Acessaram o serviço de saúde mental durante a pandemia \\
\hline Saúde da Família & $83,3 \%$ \\
Saúde do Adulto & $61,9 \%$ \\
Saúde Mental & $54,2 \%$ \\
Multiprofissional em Atenção Hospitalar & $23,5 \%$ \\
Gestão Hospitalar & $0 \%$ \\
\hline
\end{tabular}

Fonte: Autores.

\section{Discussão}

\subsection{Perfil socioeconômico, demográfico e profissional}

Em relação ao perfil socioeconômico, demográfico e profissional, os achados desse estudo vão ao encontro dos dados obtidos por uma pesquisa realizada sobre fatores associados à ansiedade entre residentes multiprofissionais em saúde durante a pandemia da COVID-19, que mostrou que a maioria dos residentes multiprofissionais eram mulheres com faixa etária acima de 20 anos, com distribuição de frequência igualitária entre receber apenas a bolsa da residência e receber entre quatro a dez salários mínimos, sendo que 59,70\% não sentia segurança técnica e científica para prestar cuidados aos pacientes COVID-19 (Dantas et al., 2020).

O presente estudo indicou que a maioria dos residentes eram do sexo feminino, com idade média de 26 anos, viviam com a família e com companheiro(a), possuíam dedicação exclusiva ao serviço de residência, tinham renda familiar entre 3 a 4 salários mínimos, excluindo os valores financeiros da bolsa de residência, e não moravam com alguém considerado como grupo de risco para COVID- 19.

Esse cenário permitiu inferir que os impactos na saúde mental dos residentes, foram provenientes, em grande parte, à natureza da atividade laboral destes, e que essas consequências podem ter sido minimizadas devido ao suporte emocional e material que esses profissionais tiveram nas suas moradias. Cabe destacar a bonificação concedida pelo Ministério da Saúde, que proporcionou uma segurança financeira aos residentes, por meio do programa "Brasil Conta Comigo", o que configura um fator protetivo para estes (Brasil, 2021).

Tais condições são corroboradas pelos achados de estudiosos, os quais demonstram que a convivência com familiares ou companheiro(a) podem trazer uma segurança afetiva e financeira, constituindo fatores protetivos para a saúde mental durante a pandemia (Ferreira et al., 2020).

Nesse sentido, a adoção de estratégias de enfrentamento adaptativas parece proteger os residentes dos riscos associados às atividades exercidas (Souza \& Araújo, 2018). Assim, torna-se essencial o desenvolvimento de estratégias práticas de saúde mental que ajudem a lidar com os transtornos que o novocoronavírus causou e está causando (OLIVEIRA, 2020). 
Todavia, cabe destacar que para a população em geral, a idade é um fator de predição para o desenvolvimento de sintomas relacionados ao estresse, depressão e ansiedade. Quanto menor a faixa etária, maior a probabilidade de desenvolvimento dos sinais associados a esses sofrimentos psíquicos, sendo a idade em torno de 30 anos, considerada a mais crítica (Ferreira et al., 2020).

Os participantes da presente pesquisa apresentaram idade média de 26 anos, tendo a máxima de 33 anos, o que significa que o aumento dos sintomas de sofrimento mental também guarda uma relação com a faixa etária dos residentes, conforme apontado pelo estudo citado acima.

\subsection{Autopercepção de saúde}

Em situações cotidianas nos serviços de saúde, os residentes da área de saúde situam-se no intervalo entre a graduação e a inserção profissional no mercado de trabalho em saúde, exigindo destes uma conciliação do processo ensino-aprendizagem com as atividades profissionais, o que, muitas vezes, pode causar sobrecarga de trabalho e ansiedade nestes.

Os fatores desafiadores no cotidiano da formação dos residentes são a sobrecarga de atividades e a escassez de articulação entre teoria e prática (Silva \& Moreira, 2019).

Acrescenta-se a esse contexto, a extensa carga horária das atividades laborais, em torno de 60 horas semanais, o que contribui ainda mais para o esgotamento mental e físico. Isto tende a agravar na emergência de uma crise sanitária, como o caso da pandemia de COVID-19.

Um estudo realizado com discentes de uma Instituição Federal de Ensino (IFE) indicou uma forte correlação entre ansiedade, depressão e estresse. Estes estudantes relataram um aumento desses sintomas no período pandêmico, quando comparado a períodos em que não havia pandemia (Esteves et al., 2021).

O novo cenário das residências exige dos residentes: raciocínio clínico; atuação nos mais variados contextos; sendo necessários destes maiores esforços físicos e psíquicos, uma vez que fazem parte de um período de transição entre estudante e profissional (Dantas et al., 2020).

Nessa pandemia, os profissionais residentes tiveram que se reinventar estabelecendo, além das atribuições diárias junto às instituições, o levantamento das demandas, a elaboração, execução e fortalecimento de ações de promoção de saúde para com os usuários dos serviços (Rebouças et al., 2020).

O cenário pandêmico trouxe à tona uma conjuntura de risco, de restrições e de comprometimento econômico e social, influenciando a saúde física e mental da população, principalmente dos profissionais de saúde e dos usuários dos serviços.

Anteriormente a pandemia, os residentes multiprofissionais em saúde, de uma IFE, das áreas de saúde mental e saúde da família já apresentavam, de forma geral, maior vulnerabilidade na área psicológica (Cahú et al., 2014).

O presente estudo apontou que ocorreu um agravamento significativo na percepção da saúde física e mental pelos residentes, no decorrer da pandemia durante o ano de 2020.

Isto pode ser confirmado pelo levantamento realizado sobre as demandas ocupacionais dos profissionais residentes da ênfase Saúde da Família e Comunidade, integrantes de um programa de Residência Multiprofissional na Atenção Primária à Saúde (APS), que revelou mudanças causadas na rotina de trabalho devido à pandemia e o surgimento de queixas ocupacionais, antes não encontradas nos cenários de prática em questão, tais como: trapezalgia, dores musculares nas regiões cervical e lombar, cansaço, ansiedade, medo e estresse (Tavares et al., 2020). Sabe-se que os profissionais da saúde costumam vivenciar situações de estresse no cotidiano dos serviços de saúde.

A incidência de síndrome de burnout ou síndrome do esgotamento profissional é significativa entre trabalhadores da saúde, e tem impacto negativo nos âmbitos pessoal, institucional, governamental e no cuidado com os usuários dos serviços (Jarruche \& Mucci, 2021). No Brasil, essa síndrome é considerada doença relacionada com o trabalho, e está presente na lista 
de transtornos mentais e de comportamento associados ao trabalho (Brasil, 1999).

Na COVID-19, houve mudanças frequentes nos protocolos de atendimento, no gasto significativo de tempo do cotidiano para a colocação e remoção dos Equipamentos de Proteção Individual (EPI) e na impossibilidade destes profissionais em interagir com outras pessoas, gerando uma sensação de exaustão e de isolamento relacionados ao processo de trabalho (Schmid et al., 2020).

Essas transformações ocorridas impactaram direta ou indiretamente na saúde física e mental dos residentes, podendo causar sofrimento mental e comprometer a assistência prestada.

Os residentes multiprofissionais em saúde devem possuir uma atenção redobrada na prestação dos cuidados assistenciais aos usuários dos serviços, no uso adequado dos EPI e no cumprimento de todos os protocolos institucionais. Assim, a prevalência de ansiedade entre os residentes, principalmente nos níveis moderado e grave, podem gerar prejuízos psicossociais e laborais nestes profissionais. Esses residentes também tiveram a saúde mental prejudicada durante a pandemia e buscaram ajuda para o controle da ansiedade (Dantas et al., 2020).

Isto corrobora com os achados do presente estudo, no qual as análises revelaram maior prevalência da ocorrência de alterações fisiológicas entre aqueles residentes que não realizam acompanhamento em saúde mental, com resultados significativos, demonstrando também que o acompanhamento de saúde mental foi fator protetor para essas alterações. Logo, o apoio psicossocial aos profissionais de saúde torna-se de fundamental importância, principalmente quando se leva em consideração o cenário pandêmico.

As práticas de apoio social mais importante são o envolvimento da liderança com os demais trabalhadores da equipe de saúde por meio de uma comunicação eficiente, do reconhecimento da importância do trabalho em equipe, da oferta de estratégias que promovam e protejam a saúde mental e promoção da autonomia do trabalhador, entre outras ações (Moreira et al., 2020).

O investimento nas ações direcionadas para a equipe médica e de enfermagem, principalmente durante o enfrentamento das epidemias, seja por meio de aconselhamento presencial ou plataformas digitais, torna-se essencial para proteger a saúde mental desses trabalhadores a curto e longo prazo (Paiano et al., 2020).

Nessa perspectiva, é imprescindível que os profissionais de enfermagem busquem estratégias de enfrentamento, tais como: apoio psicológico especializado, atendimento por telefone, realização de práticas integrativas complementares, e de exercícios de relaxamento, procura por serviços públicos de saúde mental disponíveis, para obterem melhoria das suas condições de trabalho, e de sua saúde física e mental (Dal'Bosco et al., 2020).

Assim, as organizações e as instituições devem fornecer acesso rápido a aconselhamento, a atendimento psiquiátrico e a contingência para afastamento do trabalho aos profissionais que necessitarem, bem como observarem o cumprimento das jornadas de trabalho e das folgas, essenciais para o bem-estar da equipe (Brito et al., 2020).

O cuidado integral ao profissional de saúde em sofrimento e a escuta atenta quanto à relação deste com o trabalho podem constituir importantes ferramentas a fim de evitar a medicalização desnecessária das questões que poderiam ser aliviadas e/ou prevenidas, pois se o trabalhador acumula suas ansiedades, estresse e tensões, a tendência é que ele adoeça (Tavares et al., 2020).

Cabe destaque, na presente pesquisa que os residentes das áreas de Saúde da Família, Saúde do Adulto e Saúde Mental foram os que mais procuraram o serviço de saúde mental durante a pandemia. Isto foi devido à apropriação de uma formação mais humanizada e crítico-reflexiva, bem como ao perfil do profissional, com ênfase no conceito ampliado de saúde e na integralidade das ações.

Um estudo sobre conceito ampliado de saúde na formação dos profissionais de educação física indicou um maior conhecimento sobre esse conceito pelos profissionais atuantes na área da saúde. Tal fato pode ser explicado pela participação 
profissional ativa no campo da Saúde Coletiva, em que as diretrizes e os princípios do Sistema Único de Saúde (SUS) se fazem presentes (Grillo et al., 2010).

Além disso, todo o processo formativo da residência proporciona aos residentes um olhar biopsicossocial do adoecimento e das demandas inerentes a este (Rebouças et al., 2020).

Infere-se que o fato dos residentes da área de Gestão Hospitalar não procurarem o serviço de saúde mental pode ser explicado pela própria formação destes durante as graduações de Administração e Economia, nas quais não há uma aproximação com esta temática, cabendo aos programas de residências sanar essa lacuna, com vistas à qualidade da assistência em saúde

\section{Limitações do Estudo}

Devido às instruções sanitárias de isolamento social advindas da pandemia, fez-se necessário o preenchimento on-line do questionário, o que pode ter ocasionado uma menor adesão dos participantes, visto que dos 99 residentes que atenderam aos critérios de elegibilidade, 47 não participaram da pesquisa.

Além disso, um outro motivo para essa menor adesão pode ter sido a sobrecarga de trabalho durante a pandemia, uma vez que, a carga horária semanal foi mantida (60 horas: 48 horas de prática no cenário e 12 horas de carga teórica), com sobrecarga das atividades relativas aos cuidados/atenção próprios da situação criada pela pandemia.

\section{Considerações Finais}

A pandemia de COVID-19 permitiu sensíveis mudanças na área da Saúde Coletiva ao propiciar ainda mais o protagonismo dos profissionais de saúde e a valorização do Sistema Único de Saúde. As Residências em Saúde ao possibilitarem aos residentes um olhar diferenciado sobre o processo saúde-doença, ressignificaram o papel destes na pandemia, criando vínculos, além de proporcionar condições para o empoderamento dos residentes. Esses residentes nessa pandeia vieram conquistando espaços, e se destacaram no cuidado em saúde, visando à melhoria da qualidade da assistência prestada aos usuários dos serviços e ao fortalecimento do SUS.

Nesse contexto, ao se levar em consideração "o cuidar de quem cuida", esse trabalho mostrou a interface existente entre saúde mental e pandemia da COVID-19 em um grupo de profissionais de saúde, uma vez, os residentes perceberam mudanças significativas na sua saúde física e mental durante o período pandêmico, sendo o acompanhamento de saúde mental um fator protetor para as alterações fisiológicas. Todavia, torna-se necessário avançar no sentido de adquirir resiliência, ou seja, capacidade para se adequar às mudanças impostas pela crise sanitária.

No presente estudo, não foi possível caracterizar como ocorre o acesso aos serviços de saúde mental pelos residentes. Este aspecto poderá ser abordado em estudos futuros, que discutam as políticas institucionais para esses profissionais, subsidiando os gestores para a tomada de decisão no tocante à saúde destes.

\section{Referências}

Brasil (1999). Ministério da Saúde. Portaria $n^{o} 1339$. Institui a lista de doenças relacionadas ao trabalho, a ser adotada como referência dos agravos originados no processo de trabalho no Sistema Único de Saúde, para uso clínico e epidemiológico, constante no Anexo I desta portaria. https://bvsms.saude.gov.br/bvs/saudelegis/gm/1999/prt1339_18_11_1999.html

Brasil (2021). Ministério da Saúde. Portaria $n^{\circ}$ 580. Dispõe sobre a Ação Estratégica "O Brasil Conta Comigo - Residentes na área de Saúde", para o enfrentamento à pandemia do coronavírus (COVID-19). https://www.in.gov.br/en/web/dou/-/portaria-n-580-de-27-de-marco-de-2020-250191376. 2020.

Brito, B. F. d. M., Sousa, A. M. d., Alencar, K. C., \& Martins, R. M. (2021). A pandemia do COVID-19 e um novo problema de saúde pública: Os impactos emocionais em profissionais de saúde. Research, Society and Development, 10(6). https://doi.org/DOI: http://dx.doi.org/10.33448/rsd-v10i6.15516

Cahú, R. A. G. et al (2014). Estresse e qualidade de vida em residência multiprofissional em saúde. Revista Brasileira de Terapias Cognitivas, 10 (2), 76-83. 
Research, Society and Development, v. 10, n. 16, e59101623134, 2021

(CC BY 4.0) | ISSN 2525-3409 | DOI: http://dx.doi.org/10.33448/rsd-v10i16.23134

Campos, I (2019). Crise, rede e hospitalidade: uma abordagem para a reforma psiquiátrica. Políticas e cuidado em saúde mental: contribuições para a prática profissional. Rio de Janeiro, Editora Fiocruz, 161-199.

Dal'Bosco, E. B. et al (2020). A saúde mental da enfermagem no enfrentamento da COVID-19 em um hospital universitário regional. Rev Bras Enferm, 73(2), 1-7.

Dantas, E. S. O. et al (2020). Fatores associados à ansiedade em residentes multiprofissionais em saúde durante a pandemia por COVI-19. Revista Brasileira de Enfermagem, 74, 327-345.

Esteves, C. S. et al (2021). Avaliação de sintomas depressivos em estudantes durante a pandemia do COVID-19. REFACS, 9, 9-17.

Ferreira, F. O. et al (2020). Coping in the Covid-19 pandemia: how diferent resources and strategies can be risk or protective factors to mental health in the Brazilian population. In: Health Psychology and Behavioral Medicine. https://www.tandfonline.com/loi/rhpb20.

Guedes, A. da C. et al (2021). A trajetória terapêutica de um usuário de saúde mental. J. nurs. Health; 10(2).

Grillo, L. P. et al (2019). Conceito ampliado de saúde na formação dos profissionais de educação física. Saúde e pesquisa, 12, 265-273.

Jarruche, L. T.; Mucci, S. (2021). Síndrome de burnout em profissionais da saúde: revisão integrativa. Rev. Bioética, 29 (1), 162-73.

Moreira, A. S. et al (2020). Apoio psicossocial e saúde mental dos profissionais de enfermagem no combate à COVID-19. Rev. Enfermagem em foco,11, 155161.

Mussi/Universidade do Estado da Bahia (UNEB), R. F. d. F., Mussi/Universidade do Estado da Bahia (UNEB), L. M. P. T., Assunção/Universidade do Estado da Bahia (UNEB), E. T. C. A., \& Nunes/Universidade do Estado da Bahia (UNEB), C. P. (2019). Pesquisa Quantitativa e/ou Qualitativa: distanciamentos, aproximações e possibilidades. SUSTINERE, 7(2), 414-430.

Oliveira, E. N. (2020). Saúde Mental durante a Pandemia do novo Coronavírus: algumas reflexões necessárias. Research, Society and Development, 9(8). https://doi.org/DOI: http://dx.doi.org/10.33448/rsd-v9i8.5478

Paiano, Marcelle et al (2020). Saúde mental dos profissionais de saúde na China durante a pandemia do novo coronavírus: revisão integrativa. Revista brasileira de enfermagem, 73, 327-345.

Pereira, A. S., Shitsuka, D. M., Parreira, F. J., \& Shitsuka, R. (2018). Metodologia da pesquisa CIENTÍFICA (1st ed.). UAB/NTE/UFSM.

Rebouças, E. R. N. et al (2020). Residência multiprofissional: contribuições durante a pandemia. Cadernos Esp. Ceará, 14,118-123.

Schechtman, A; Alves, D. S. (2019). A organização da Política de Saúde Mental. Políticas e cuidado em saúde mental: contribuições para a prática profissional. Rio de Janeiro, Editora Fiocruz, 41-58.

Schmidt, B. et al (2020). Saúde mental e intervenções psicológicas diante da pandemia do novo coronavírus (COVID-19). Estudos de Psicologia (Campinas), 37.

Silva, R. M. B.; Moreira, S. da N. T. (2019). Estresse e Residência Multiprofissional em Saúde: Compreendendo Significados no Processo de Formação. Revista Brasileira de Educação Médica, 43 (4), 157-166.

Souza, E. C. P. de; A., Tereza, C. C. F. (2018). Percepção sobre formação em residência na área da saúde: necessidades, expectativas e desafios. Rev. SBPH, $21(1)$.

Sy, A. (2018). La medicalización de la vida: hibridaciones ante la dicotomía Naturaleza/Cultura. Ciência \& Saúde Coletiva, 23,1531-1539.

Tavares, B. L. L. et al (2020). Cuidar e ser cuidado em tempos de pandemia. Cadernos Esp. Ceará, 14, 138-142.

Zangirolami-Raimundo J., Echeimberg, J. O., Leone C. (2018) Research methodology topics: Cross-sectional studies. Journal of Human Growth and Development. 28(3): 356-360. 